\title{
Mécanique et régulation de la migration kératinocytaire
}

Du fait du renouvellement permanent de l'épiderme, les kératinocytes sont soumis à un mouvement vertical de l'assise basale vers la couche cornée. Lors de la phase de réépithélialisation des plaies, ce mouvement essentiellement passif fait place à une importante mobilité latérale. Ce phénomène : la migration kératinocytaire, est sous la dépendance de nombreux facteurs, particulièrement les molécules de la matrice extracellulaire et les cytokines présentes dans le lit de la plaie. L'interaction entre les molécules matricielles (fibronectine, collagène, vitronectine, laminine, etc.) et les kératinocytes se fait au travers des récepteurs membranaires de la famille des intégrines. Cette interaction joue un rôle essentiel dans la migration kératinocytaire car elle assure tout à la fois l'adhérence entre les cellules et le substrat et la transmission aux cellules de signaux qui, comme ceux délivrés par les cytokines, contrôlent et modulent ce phénomène.

\section{Denis Jullien Yves Sarret} Catherine Stamm Daniel Schmitt

\footnotetext{
ADRESSE

D. Jullien : étudiant en thèse. Y. Sarret : chercheur post-doctoral. C. Stamm: interne, D. Schmitt: directeur de recherche, directeur de l'U. 346 de l'Inserm. Inscrm U. 346 ct clinique dermatologique, hôpital ÉdouardHerriot, pavillon R, 69437 Lyon Cedex 03, France.

es kératinocytes, quand ils participent au phénomènc de réépithélialisation, deviennent des cellules extrêmement mobiles. La connaissance de la mécanique qui autorise cette fonction et des facteurs qui la règlent est primordiale pour comprendre de multiples phénomènes cellulaires et tissulaires impliqués dans la pathologie dermatologique courante. La peau, qui couvre en moyenne une surface de $1,7 \mathrm{~m}^{2}$ chez l'adulte, est le plus volumineux compartiment de l'organisme. L'épiderme, son feuillet le plus externe, est constitué à $95 \%$ par des kératinocytes, mais seuls ceux des couches profondes, qui sont les moins différenciés, peuvent devenir lors d'une plaic des cellules latéralement mobiles et recouvrir la perte de substance (réépithélialisation). En situation physiologique, les kératinocytes basaux reposent sur une membrane basale appelée jonction dermo-épidermique (figure 1). Cette dernière, produite majoritairement par les kératinocytes basaux mais aussi par les fibroblastes du derme sous-jacent, comporte en microscopie électronique trois zones (la lamina lucida, la lamina densa et la sub-lamina densa) au sein desquelles une vingtaine de molécules sont à ce jour identifiées et se répartissent de manière diffuse ou compartimentée. Les kératinocytes basaux reposent sur la lamina lucida, zone la plus externe riche en laminine. $\mathrm{Au}$ contact de cette glycoprotéine, les kératinocytes basaux établissent des structures d'adhérence forte, appelées hémidesmosomes, les rendant immobiles latéralement. En situation de plaie, les kératinocytes basaux entrent en contact avec d'autres macromolécules comme la fibronectine, le collagène $I$, le collagène IV, la thrombospondine 


\section{RÉFÉRENCES}

1. Sarret Y, Woodley DT, Goldberg GS, Kronberger A, Wynn KC. Constitutive synthesis of a $92-\mathrm{kDa}$ keratinocyte-derived type IV collagenase is enhanced by type I collagen and decreased by type IV collagen matrices. J Invest Dermatol 1992; 99 : 836-41.

2. Mast S. Structure, movement, locomotion and stimulation in amoebae. J Morphol Physiol 1926 ; 41 : 347-425.

3. Rubino S, Fighetti M, Unger E, Capucinelli $P$. Location of actin, myosin, and microtubular structures during directed locomotion of Dictyostelium amoebae. J Cell Biol 1984 ; 98 : 282-390.

4. Allen R. A new theory of amoeboid movement and protoplasmic streaming. Exp Cell Res 1961; 8S : 17-31.

5. Fukui Y, Lynch J, Brzeska H, Korn E. Myosin is located at the leading edges of locomoting Dictyostelium amoebae. Nature 1989 ; 341 : 328-31.

6. Demma H, Dharmawardhane S, Eddy R, Hall A, Sauterer R, Warren V' Mechanism of amoeboid chemotaxis : an evaluation of the cortical expansion model. Dev Genetics 1990 ; 11 : 333-40.

7. Stossel TP. How cells crawl, with the discovery that the cellular motor contains muscular proteins, we can begin to describe cell motility in molecular detail. American Scientist $1990 ; 78: 408-23$.

8. Foscher P. Calcium and polyphosphoinositide control of cytoskeletal dynamics. Trends Neurosci $1989 ; 12$ : 474-86.

9. Theriot JA, Mitchinson TJ. Actin microfilament dynamics in locomoting cells. Nature $1991 ; 352$ : 126-31 ou l'héparan-sulfate protéoglycane qui induisent la locomotion kératinocytaire (figure 1). Lors de la migration, les kératinocytes basaux sécrètent des métalloprotéases qui participent à la détersion de la plaie [1]. Diverses cytokines comme l'EGF (epidermal growth factor), les TGF $\alpha$ et $\beta$ (transforming growth factor $\alpha$ et $\beta$ ), l'interféron $\gamma$ (IFN $\gamma$ ) peuvent être stockées au sein de la structure en réseau de la jonction dermo-épidermique. Ces cytokines favorisent la cicatrisation et sont susceptibles de moduler le phénomène migratoire. Lors d'une plaie, la perte de substance emporte l'épiderme et la jonction dermoépidermique entraînant une modifica- tion profonde du microenvironnement des kératinocytes situés au bord de la lésion. La réépithélialisation de la plaie, événement précoce lors de la cicatrisation, est alors obtenue par la conjonction de deux phénomènes : la migration et la prolifération kératinocytaire. La réépithélialisation peut être mise en défaut dans certaines pathologies cutanées fréquentes comme les ulcères de jambe ou les brûlures.

Il nous a donc semblé nécessaire de faire la mise au point des connaissances sur la migration kératinocytaire. Nous étudierons en premier lieu la mécanique cellulaire qui sous-tend cette fonction, les facteurs influençant

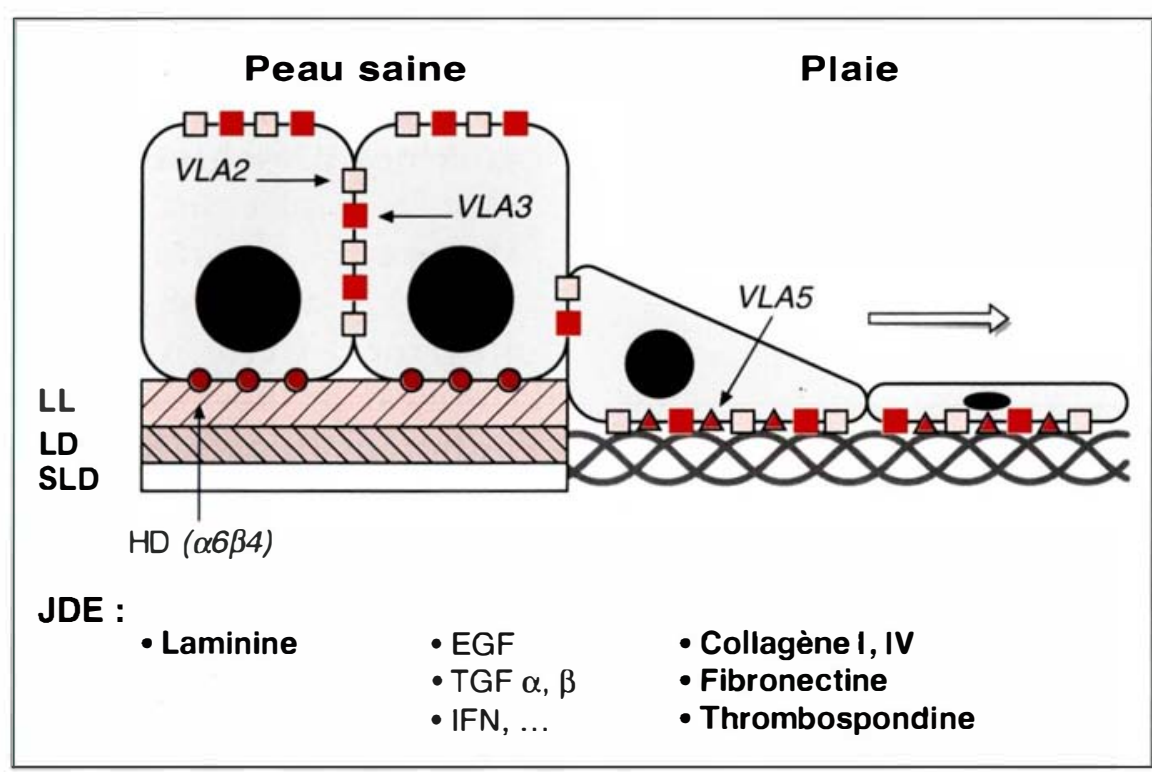

Figure 1. Rapports des kératinocytes humains basaux en peau saine et lésée. En peau saine, les kératinocytes basaux reposent sur la jonction dermo-épidermique au sein de laquelle on distingue en microscopie électronique trois zones : la lamina lucida (LL) riche en laminine, la lamina densa (LD) et la sub lamina densa (SLD). Les nombreuses macromolécules entrant dans la composition de cette membrane basale sont liées les unes aux autres et forment un réseau tridimentionnel en filet à l'intérieur duquel peuvent $s^{\prime}$ accumuler différentes cytokines (EGF, TGF $\alpha$ et $\beta$, INF $\gamma .$. ). Au contact de la jonction dermo-épidermique, les kératinocytes basaux sont immobiles, ils expriment à leur pôle inférieur des structures d'adhérence forte, les hémidesmosomes (HD) comprenant l'intégrine $\alpha 6 \beta 4$, récepteur de la laminine. Les intégrines VLA2 et VLA3, récepteurs de différentes macromolécules matricielles dont les collagènes I et $I V$, sont exprimées préférentiellement sur les faces cellulaires apicales et latérales. En situation de plaie, le microenvironnement kératinocytaire est complètement modifié. La jonction dermo-épidermique est détruite et laisse place à un nouvel environnement matriciel servant de support à la migration kératinocytaire. Les collagènes I et IV, et la fibronectine entrent au contact des kératinocytes basaux du bord de la plaie. Ces derniers voient alors leur morphologie se modifier, la distribution de VLA2 et VLA3 n'est plus restreinte aux faces cellulaires latérales et apicales, ils expriment VLA5, récepteur pour la fibronectine, et migrent pour recouvrir la perte de substance. Ce phénomène associé à la multiplication des kératinocytes basaux correspond à la phase de cicatrisation appelée réépithélialisation. 
la migration et enfin les implications thérapeutiques découlant de ces connaissances.

\section{Modèles}

de migration cellulaire

Les mécanismes moléculaires qui supportent la motricité cellulaire sont loin d'être compris. Les cellules les plus utilisées comme modèles d'étude sont l'amibe Dictyostelium amoebae, les fibroblastes, les leucocytes et des cellules tumorales. En l'absence de données spécifiques concernant les kératinocytes basaux, nous essaierons de dresser une esquisse de ce que peut être leur mécanique motrice au travers des résultats recueillis sur ces autres types cellulaires.

Les kératinocytes basaux se déplacent en étendant leur corps cellulaire dans le sens du mouvement et en le rétractant au pôle opposé. Différentes molécules d'adhésion, comme les intégrines de la famille VLA (very late activation antigen), participent à l'établissement de liaisons transitoires entre la cellule et son substrat, créant les contraintes nécessaires à l'apparition du mouvement. Le déplacement se fait de manière aléatoire ou orientée en fonction du mécanisme qui l'induit. Dans le premier cas, qui correspond au phénomène de chémokinésie, on observe à des temps donnés et sur toute la circonférence cellulaire des zones où se forment des prolongements cellulaires et des zones où le corps cellulaire se rétracte. Le déplacement orienté se subdivise en haptotaxie (gradient de macromolécules matricielles) et en chimiotaxie (gradient de molécules solubles). Lors du déplacement orienté, qui est le modèle du déplacement des kératinocytes basaux en situation de plaie, il y a polarisation de la cellule, les prolongements cellulaires se formant au front de migration tandis que le pôle opposé se rétracte. Ainsi, la manifestation morphologique la plus caractéristique d'un kératinocyte en mouvement, observé en microscopie optique, est l'existence à son bord antérieur, par rapport au front de migration, d'une région cytoplasmique élargie et aplatie appelée lamelle à l'extrémité de laquelle sont émis soit de très minces feuillets de cytoplasme, les lamellipodes, soit de fins prolon$\mathrm{m} / \mathrm{s} n^{\circ} 4$ vol. 9, avril 93 gements filamenteux, les filopodes, qui entrent en contact avec le substrat pour créer de nouveaux points d'adhérence (figure 2). De nombreux auteurs ont fait de ces structures les acteurs principaux de la migration et plusieurs théories tentent d'expliquer leur formation.

La plus ancienne (figure 3A) [2] repose sur l'existence d'une force contractile située au pôle postérieur de la cellule qui, en poussant la partie fluide du cytoplasme (l'endoplasme) vers l'avant au travers de la partie corticale visqueuse (l'ectoplasme), donne naissance aux lamellipodes dont la forme est fixée par évolution vers une texture de type ectoplasmique. La mise en évidence de myosine II et d'actine au pôle postérieur de multiples cellules [3] et la description du phénomène de polymérisation des monomères d'actine entraînant une augmentation de la viscosité cytoplasmique dans les lamellipodes sont en faveur de cette hypothèse. Le modèle dit de fluidisation/contraction est une transposition de cette théorie et cherche à expliquer le mouvement de la totalité du corps cellulaire. Selon ce modèle, la contraction du cortex d'actomyosine au pôle postérieur dc la cellule pousse vers l'avant le cytoplasme, et ce dernier, alors qu'il était

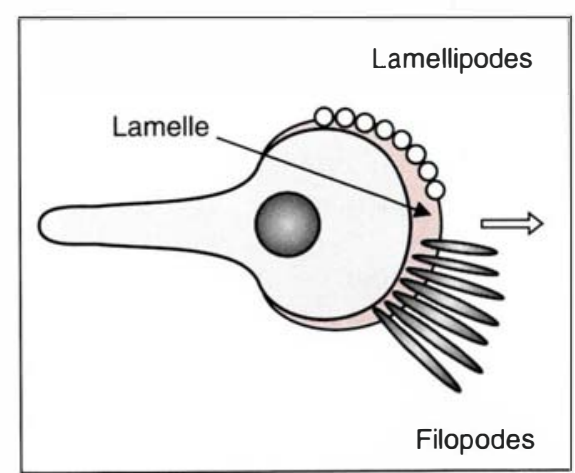

Figure 2. Kératinocyte en mouvement. La lamelle est une fine lame cytoplasmique aplatie présente au pôle antérieur de la cellule. En émergent soit de fins feuillets cytoplasmiques, les lamellipodes, soit de fins prolongements cytoplasmiques, les filopodes. Ces deux structures permettent au corps cellulaire de se propulser vers l'avant, d'établir de nouveaux contacts avec le substrat. Ce phénomène, associé à la rétraction du corps cellulaire au pôle postérieur de la cellule, permet à la cellule d'avancer. fluide au pôle postérieur de la cellule, prend, au pôle antérieur, une structure gélifiéc. Il y a protrusion du bord antérieur de la cellule, formation de nouvelles liaisons avec le substrat tandis qu'au pôle postérieur de la ccllule ces liaisons se détachent, permettant au corps cellulaire de se rétracter. La répétition de cette séquence de phénomènes engendre un mouvement antérograde.

Une deuxième théorie visant à expliquer la formation des filopodes/lamellipodes est fondée sur l'hypothèse qu'au sein même de ces structures existe une force de glissement qui, en propulsant les composants du cytoplasme vers l'avant, repousse la membrane plasmique (figure 3B) [4]. Cette théorie d'un lamellipode " autopropulsé " s'accorde avec l'observation de lamellipodes migrant bien qu'ils soient détachés de leurs corps cellulaires. La myosine II étant absente des lamellipodes, ce serait la myosine I qui pourrait fournir le support moléculaire nécessaire à la génération de la force de glissement [5]. La théorie de l'extension corticale (figure 3C) [6] propose une troisième origine à la force requise pour la prorusion des lamellipodes et des filopodes. Ellc est particulièrement intéressante pour expliquer la réponse localisée nécessaire à la protrusion orientée dans les phénomènes d'haptotaxie ou de chimiotaxie. En résumé, en réponse à un signal extracellulaire local, il y a activation de la voie des polyphospho-inositides et régulation de la fonction de différentes protéines intracytoplasmiques comme la gelsoline et la profiline. Il s'ensuit dans un premier temps une dépolymérisation locale du cortex d'actine entraînant une augmentation ponctuelle de l'osmolarité. Cette hyperosmolarité entraîne une mobilisation de l'eau vers cette région, ce qui crée une force qui repousse la membrane plasmique. Dans un second temps, une repolymérisation de l'actine sous forme de filaments est permise grâce à des molécules comme la plastine, l' $\alpha$-actinine ou l'actin binding protein donnant naissance à différentes structures cytosquelettiques. Ainsi, les lamellipodes sont stabilisés par un réseau tridimentionnel de filaments d'actine alors qu'au sein des filopodes, on trouve des faisceaux 
de filaments d'actine [7, 8]. Dans des hératinocytes de poisson où l'essentiel du cytosquelette d'actine est situé dans un large et unique lamellipode antérieur, le mouvement cellulaire est directement lié à la formation de nouveaux filaments d'actine au front de migration [9].

A l'intérieur des lamellipodes de la majorité des cellules étudiées, il existe un flux rétrograde de molécules d'actine qui est le reflet du mouvement centripète, sous forme de filaments, des monomères d'actine polymérisés dans la zone du front d'avancement (figure 3D) [10]. A la base des lamellipodes, les filaments d'actine sont rompus, autorisant le recyclage des monomères. Ce flux centripète de filaments d'actine pourrait fournir une force de traction s'il existait un ancrage au moins transitoire du cytosquelette d'actine à la matrice sous-jacente. Une telle fonction peut ctre remplie par les foyers riches en intégrines et en taline qui sont présents au bout des câbles de filaments d'actine dans les lamellipodes [11]. En effet, les intégrines sont des hétérodimères transmembranaires qui pcuvent, par leur fragment cytoplasmique, se lier à l'actinc via des composants comme la taline, l' $\alpha$-actinine ou la vinculine et, par leur fragment extracellulaire, s'attacher spécifiquement à une grande variété de macromolécules matricielles.

La zone lamellaire situéc en arrière des lamellipodes contient un réseau cortical de filaments d'actine ainsi que des faisceaux de filaments d'actine associés à de la myosine II appelés fibres de contrainte (figure $3 E$ ). Ces dernières se forment au niveau des points focaux qui sont des structures d'adhérence fortc de la cellule au substrat et qui sont constitués, notamment, d'une concentration de différents types d'intégrines, de taline, d' $\alpha$-actinine et de vinculine [12]. Les fibres de contrainte sont dirigées vers l'arrière de la zone lamellaire et sont désassemblées à sa base dans la région périnucléaire. Si elles peuvent se contracter, appliquer une tension sur le substrat et donc engendrer le mouvement, on doit cependant noter que leur nombre, comme celui des points focaux, est inversement corrélé à l'efficacité de la locomotion. Cette observation est à rapprocher de celle faite sur des cellules embryonnaires, montrant qu'cn phase migratoire la distribution de l'intégrine $\alpha 5 \beta 1$ est diffuse à la surface cellulaire et le cytosquclettc désorganisé, alors qu'en phase stationnaire $\alpha 5 \beta 1$ est engagée dans des points focaux d'où naissent des structures cytosquelettiques organisées [13].

Une autre théorie repose sur l'observation de l'existence d'un flux rétrograde continu de composants corticaux du cytosquelette sur des cellules en cours de migration (figure 3F) [14]. Lc réseau de filaments d'actine et de myosine II, qui n'est présent qu'à la partie centrale et postérieure de la cellule, est en ćtat de contraction permanent. Cette tension le tire vers l'arrière de la cellule, entraînant un flux rétrograde continu d'actomyosine. Au pôle postérieur de la cellule, le réseau est désassemblé pour ĉtre recyclé. Le couplage de ce réseau au substrat permet de créer une force de sens opposé qui propulse la ccllule vers l'avant.

Pour cc dernicr modèle, comme pour tous ccux envisagés précédemment, la production d'unc force ne débouchera sur un mouvement que si elle s'excrce par rapport au substrat environnant, c'est-à-dire uniquement si la ccllulc peut se fixcr de manière réversible à celui-ci. Nous envisagerons plus loin la part prise par les intégrines dans cettc fonction.

$\mathrm{Si}$ les théorics que nous venons d'envisager semblent multiples, il est en fait vraisemblable qu'il y ait redondance des systèmes autorisant un phénomène aussi fondamental que la migration ccllulaire. Le mouvement résulterait alors d'une combinaison de certains des mécanismes envisagés, la prépondérance de l'un ou l'autre permettant une réponse adaptée à la situation comme par exemple l'extension polarisée de lamellipodes et de filopodes au contact d'une molécule attractante.

\section{Facteurs modulant la migration kératinocytaire (Tableau I)}

Parmi les différentes techniques étudiant la migration cellulaire, seule l'utilisation d'une caméra vidéo 


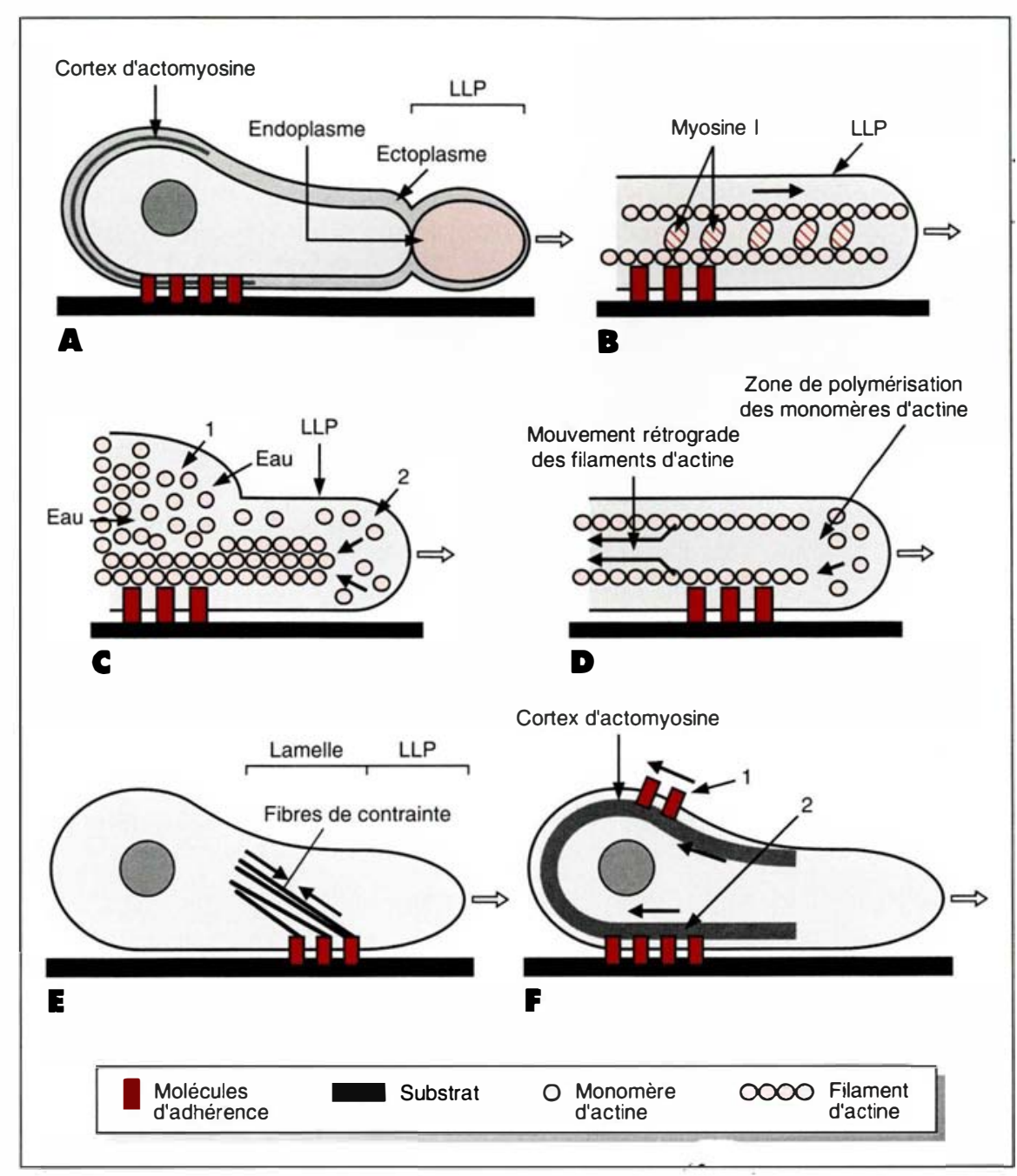

Figure 3. Modèles de migration cellulaire. (A) La contraction du cortex d'actomyosine à la partie postérieure de la cellule pousse vers l'avant l'endoplasme au travers de l'ectoplasme. II y a formation d'un lamellipode (LLP) et donc protrusion du corps cellulaire vers l'avant. (B) Les molécules de myosine I permettent le glissement des filaments d'actine les uns par rapport aux autres, créant une force qui repousse le bord antérieur de la cellule. Il y a formation d'un LLP et donc protrusion du corps cellulaire vers l'avant. (C) En réponse à un signal extracellulaire local, il y a dépolymérisation du cortex d'actine (1) ce qui entraîne une augmentation locale de l'osmolarité et un appel d'eau, créant une force qui repousse la membrane plasmique. Dans un second temps, la repolymérisation des monomères $d^{\prime}$ actine sous forme de filaments (2) aboutit à stabiliser le LLP ainsi formé. (D) La polymérisation des monomères $d^{\prime}$ actine au bord antérieur de la cellule s'accompagne d'un flux rétrograde des filaments $d^{\prime}$ actine ainsi formés. L'existence $d^{\prime}$ une liaison entre ces filaments et le substrat, au travers de molécules transmembranaires, transforme ce mouvement rétrograde en mouvement antérograde du corps cellulaire. (E) Les fibres de contrainte sont susceptibles de se contracter. Elles transmettent une contrainte au substrat au travers des molécules transmembranaires présentes dans les points focaux. II s'ensuit un déplacement antérograde du corps cellulaire. (F) Le cortex d'actomyosine présent uniquement à la partie centrale et postérieure du corps cellulaire est en état de contraction permanent créant un flux rétrograde continu d'actomyosine. Au pôle postérieur de la cellule, il est dissocié et ses éléments sont recyclés. Les protéines transmembranaires, quand elles sont liées aux éléments de ce cortex, se déplacent avec lui vers l'arrière de la cellule (1). En revanche, si elles sont en plus liées au substrat (2), une force s'exerce sur celui-ci et débouche sur un mouvement antérieur du corps cellulaire. autorise l'étude directe sans interférences de l'effet d'une molécule sur le mouvement cellulaire. Le coût de cet équipement et son inaptitude à étudier de grands échantillons ont conduit au développement de procédés alternatifs qui souvent, hélas, observent d'autres données que la seule migration. C'est le cas des techniques mesurant l'avancement du front de migration à partir d'explants tissulaires pour lesquelies la multiplication cellulaire est un biais considérable. Les techniques comme l'agarose drop explant nécessitent que les cellules tolèrent l'agarose. Le phagokinetic track assay (figure 4), [15] et les modèles dérivés de la chambre de Boyden sont pour l'heure les meilleures alternatives à l'utilisation d'une caméra vidéo.

Les macromolécules présentes dans la jonction dermo-épidermique et celles susceptibles d'entrer en contact avec les kératinocytes lors d'une plaie ont été particulièrement étudiées in vitro. La laminine inhibe de manière dépendante de sa concentration la migration induite par d'autres macromolécules comme le collagène IV, cet effet nécessite la structure intacte de la molécule native [16]. Le collagène IV, qui dans la jonction dermoépidermique est localisé dans la lamina densa à distance des kératinocytes basaux, stimule considérablement la migration (figure 4), et cet effet semble indépendant de la structure tertiaire de la molécule [16]. Le collagène I, présent dans le derme, a un très important effet inducteur sur la migration kératinocytaire, la qualité de cet effet étant liée au maintien de la structure en triple hélice [16]. La migration kératinocytaire est aussi induite par la fibronectine [17] et c'est le fragment d'attachement cellulaire de la molécule qui relaie cette fonction, au moins partiellement, au travers d'une séquence RGD [18]. L'effet de la vitronectine sur la migration est très faible et semble en fait surtout lié au phénomène d'étalement cellulaire. La vitronectine n'induit pas de motricité directionnelle comme peuvent le faire la fibronectine ou les collagènes I et IV. Elle inhibe, quand elle lui est associée, l'effet dû au collagène [19]. La thrombospondine possède, elle aussi, un effet favorisant sur la migration 


\section{RÉFÉRENCES}

19. Brown C, Stenn KS, Falk RJ, Woodley DT, O'Kecfe EJ. Vitronectin : effects on keratinocytc motility and inhibition of collagen-induced motility. $J$ Invest Dermatol 1991 ; 96 : 724-8.

20. Nickoloff BJ, Mitra RS, Risr BL, Dixit VM, Varani J. Modulation of keratinocytc motility. Correlation with production of extraccllular matrix molecules in responsc to growth promoting and antiproliferative factors. Am J Pathol 1988 ; 132 : 543-50.

21. Donaldson DJ, Mahan JT. Fibrinogen and fibronectin as substrates for cpidermal ccll migration during wound closurc. $J$ Cell Sci $1983 ; 62$ : 117-27.

22. Barrandon Y, Green H. Cell migration is cssential for sustained growth of keratinocytc colonics : the roles of transforming growth factor- $\alpha$ and cpidermal growth factor. Cell 1987 ; 50 : 1131-7.

23. Sarret $Y$, Woodlcy DT, Grigsby $K$, Wynn KC, O'Kcefe EJ. Human keratinocyte locomotion : the effect of sclected cytokincs. J Invest Dermatol 1992 ; 98 : 12-6.

24. Stoker M, Gherardi E, Perryman M, Gray J. Scatter factor is a fibroblast-derived regulator of cell mobility. Nature $1987 ; 327$ : 239-42.

25. Matsumoto K, Hashimoto K, Yoshikawa K, Nakamura T. Marked stimulation of growth and motility of human kcratinocytes by hepatocytc growth hormonc. Exp Cell Res 1991; 196 : 114-20.

26. Chen JD, Kim JP, Sarret Y, et al. Epidermal growth factor (EGF) promotes human keratinocytc locomotion via enhanced cxpression of relcvant integrin subunits

kératinocytaire, mais, selon la technique utilisée pour le mettre en évidence, il est faible ou très important [20]. Le collagène $\mathrm{V}$ ct l'héparan-sulfate protéoglycane ont, comme la vitronectine, un effet très faible essentiellement dû à l'accroissement des mouvements liés au phénomène d'attachement [16]. Le fibrinogène favorise la migration des kératinocytes [21].

L'effet de plusieurs cytokines a aussi été exploré. Ainsi, le TGF $\beta$ a une action positive sur la migration kératinocytaire, mais elle est essentiellement indirecte et résulte d'une augmentation de la sécrétion de fibronectine par les kératinocytes basaux [20]. Barrandon et Green ont montré qu'EGF stimulait la migration kératinocytaire [22]. En présence d'EGF, le rayon d'une colonie de kératinocytes en culture peut devenir huit fois plus important qu'en l'absence d'EGF. Cette action nécessite non seulement une stimulation de la prolifération des kératinocytes, mais, pour grandir, les éléments de la colonie doivent aussi migrer. Cette interprétation est d'autant plus probable que l'action de l'EGF est détectée dès la $15^{\mathrm{e}}$ minute, pour être maximale à 3 heures. Ce résultat est trop rapide pour dépendre d'un simple effet prolifératif [22]. L'action stimulante d'EGF sur la migration kératinocytaire passe, en partie, par l'augmentation de production - par les kératinocytes basaux - de fibronectine et de thrombospondine [20, 23]. Le TGF $\alpha$ partage le même récepteur cellulaire que l'EGF, il n'est donc pas surprenant que son effet sur la migration kératinocytaire soit de même type. Barrandon et Green ont démontré, en utilisant la même méthodologie des mégacolonies, que TGF $\alpha$ avait une action stimulante sur la migration kératinocytaire supérieure à celle d'EGF [22]. La somatomédine $\mathrm{C}$ (ou IGF2) stimule la production de thrombospondine par les kératinocytes humains et

\begin{tabular}{|l|l|l|}
\hline \multicolumn{3}{|c|}{ Tableau I } \\
MACROMOLÉCULES MATRICIELLES ET CYTOKINES PORTEUSES \\
D'UN LA MIGRATION KÉRATINOCYTAIRE \\
\hline Facteur & \multicolumn{1}{c|}{ Action } & \multicolumn{1}{c|}{ Références } \\
\hline Collagènes I et IV & +++ & {$[16]$} \\
\hline Collagène V & + & {$[16]$} \\
\hline Fibrinogène & + & {$[21]$} \\
\hline Fibronectine & ++ & {$[17,21]$} \\
\hline Héparan-sulfate protéoglycane & + & {$[16]$} \\
\hline Laminine & --- & {$[16]$} \\
\hline Thrombospondine & $+->++$ & {$[20]$} \\
\hline Vitronectine & $+1-$ ) & {$[19]$} \\
\hline Epidermal growth factor & + & {$[20,22,23]$} \\
\hline Extrait pituitaire bovin & + & {$[23]$} \\
\hline Hepatocyte growth factor & + & {$[25]$} \\
\hline Interféron $\gamma$ & + & {$[20]$} \\
\hline Scatter factor & + & {$[20]$} \\
\hline Somatomédine C & + & $20,23]$ \\
\hline Transforming growth factor $\alpha, \beta$ & + \\
\hline
\end{tabular}
Exp Cell Res 1993 (sous presse). 
leur migration [20]. L'IFN $\gamma$ inhibe la production de fibronectine et de thrombospondine par les kératinocytes humains ainsi que leur aptitude à migrer [20]. Le scatter factor (SF) est une protéine sécrétée notamment par les fibroblastes embryonnaires, ceux de certaines lignées et les cellules musculaires lisses de l'aorte. Il possède un effet promoteur sur la migration des kératinocytes [24]. L'HGF (hepatocyte growth factor), qui a lui aussi un effet favorisant sur la migration des kératinocytes [25], possède une très grande homologie de séquence avec le SF. L'extrait pituitaire bovin, qui entre dans la composition de nombreux milieux de culture, contient un ou plusieurs agents qui stimulent la migration des kératinocytes [23]. En dehors du rôle qu'elles peuvent avoir au travers des macromolécules qu'elles font sécréter, des cytokines comme l'EGF ou le SF sont susceptibles d'agir via leurs récepteurs, en augmentant par exemple l'expression à la surface cellulaire des intégrines [26] ou en activant la voie dẹs polyphospho-inositides.

L'ion calcium inhibe la migration kératinocytaire induite par différentes matrices comme les collagènes I et IV, et la fibronectine. Cet effet se manifeste très rapidement quand on élève la concentration calcique du milieu de 0,1 à $1,1 \mathrm{mM}$ et s'accompagne d'une surexpression globale des VLA intégrines [27].

\section{Intégrines et migration kératinocytaire (Tableau II)}

Deux types d'interactions entre la cellule et son environnement sont essentiels lors de la migration kératinocytaire: la réception spécifique du signal inducteur et l'adhérence réversible et labile de la cellule sur son substrat.

Les intégrines peuvent supporter cette double fonction, ce qui en fait des molécules clés de la migration. Elles forment une famille d'au moins 20 hétérodimères, présents à la surface cellulaire, et constitués d'au moins huit sous-unités $\beta$ et 14 sousunités $\alpha$ différentes. Seules six ont, à ce jour, été décrites à la surface des kératinocytes humains $(\alpha 1 \beta 1, \alpha 2 \beta 1$, $\alpha 3 \beta 1, \alpha 5 \beta 1, \alpha 6 \beta 4, \alpha \mathrm{v} \beta 5$ [28]. Leur domaine extracellulaire est porteur de la spécificité de reconnaissance avec le ligand qui, pour les intégrines kératinocytaires, peut être les collagènes I ou IV, la fibronectine, la laminine, la vitronectinc ou l'épiligrine. Il permet l'attachement de la cellule au substrat et la reconnaissance spécifique d'un signal qui peut, par exemple, induire la migration. C'est ce que l'on observe pour les collagènes I ou IV, ou la fibronectine. Le domaine

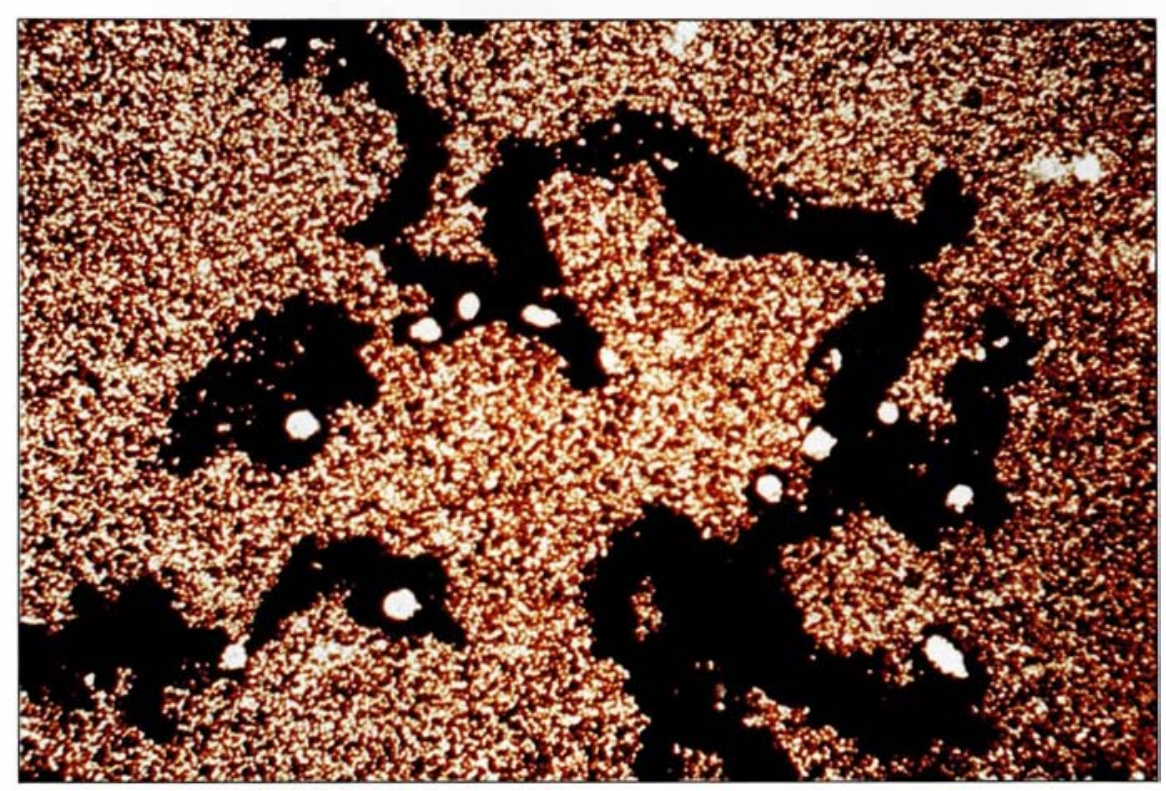

Figure 4. Phagokinetic track assay : effet du collagène IV sur la migration kératinocytaire. Kératinocytes humains ensemencés sur un tapis de grains d'or recouvert de collagène IV : les cellules (points jaunes brillants) en se déplaçant phagocytent les grains d'or et laissent derrière elles des traces noires qui permettent de quantifier la migration, qui est ici très importante.

\begin{tabular}{|c|c|c|c|c|}
\hline \multicolumn{5}{|c|}{$\begin{array}{c}\text { Tableau ॥ } \\
\text { INTÉGRINES EXPRIMÉES A LA SURFACE } \\
\text { DES KÉRATINOCYTES HUMAINS }\end{array}$} \\
\hline Intégrines & Prés. Épid. (1) & Plaie (2) & Ligand/Migration (3) & Références \\
\hline$\alpha 1 \beta 1$ & $\begin{array}{l}+++->S 15] \\
{[S 15-> \pm I-}\end{array}$ & - & Co I(?), Co IV(?), LN(?) & [28] \\
\hline$\alpha 2 \beta 1$ & IS10 $->+++$ & +++ & Co $I(+)$, Co $I V(+)$, LN(?) & {$[28,30]$} \\
\hline$\alpha 3 \beta 1$ & $<\mathrm{S} 8->+++$ & +++ & $\begin{array}{l}\text { Co } 1(-) \text {, Co } \mid V(-) \\
F N(-) \text {, LN(?), EN(?) }\end{array}$ & {$[28,30]$} \\
\hline$\alpha 5 \beta 1$ & [S14-> \pm & ++ & $\mathrm{FN}(+)$ & {$[28,30]$} \\
\hline \multirow[t]{2}{*}{$\alpha 6 \beta 4$} & $<\mathrm{S} 8->+++$ & & $L N(-), E N(?)$ & {$[28]$} \\
\hline & $\alpha v:[58-> \pm$ & $\alpha v:++$ & $F N(?), V N(?)$ & [28] \\
\hline
\end{tabular}

(1) Prés. Épid. : date d'apparition (en semaines d'embryogenèse) et taux d'expression d'une intégrine donnée dans l'épiderme humain sain.

(I : a partir de ; l : jusqu'a ; < : avant.)

(2) Plaie : expression à la surface de $\mathrm{KH}$ en situation de plaie.

(3) Ligand/migration : ligand connu de cette intégrine et rôle joué par l'intégrine lors de la migration sur ce ligand.

Co : collagène $; L N=$ laminine $; F N=$ fibronectine $; V N=$ vitronectine 


\section{RÉFÉRENCES}

27. Jullien D, Sarret Y, Lisard G, Souchier C, Schmitt D. Calcium inhibits human keratinocyte migration and enhances expression of VLA-integrins. J Invest Dermatol 1993 (sous presse).

28. Hertle MD, Adams JC, Watt FN. Integrin expression during human epidermal development in vivo and in vitro. Development $1991 ; 112$ : 193-206.

29. Chan BMC, Kassner PD, Schiro JA, Byers HR, Kupper TS, Hemler ME. Distinct cellular functions mediated by different VLA integrin $\alpha$ subunit cytoplasmic domains. Cell 1992; 68: 1051-60.

30. Kim JP, Zhang K, Kramer RH, Schall TJ, Woodley DT. Integrin receptors and RGD sequences in human keratinocyte migration : unique antimigratory function of $\alpha 3 \beta 1$ epiligrin receptor. J Invest Dermatol $1992 ; 98: 764-70$

31. Hanks SK, Calalb MB, Harper MC, Patel SK. Focal adhesion protein-tyrosine kinase phosphorylated in response to ccll attachment to fibronectin. Proc Natl Acad Sci USA 1992 ; 89 : 8487-91.

32. Ortonne JP, Löning T, Schmitt D, Thivolet $\mathrm{J}$. Immunomorphological and ultrastructural aspects of keratinocyte migration in epidermal wound healing. Virchows Arch (Pathol Anat) $1981 ; 392$ : 217-30.

33. Brown GL, Nanney LB, Griffen J, et al. Enhancement of wound healing by topical treatment with epidermal growth factor. $N$ Engl J Med 1989; 321: 76-9.

34. Schultz GS, White M, Mitchell R, et al. Epithelial wound healing enhanced by transforming growth factor- $\alpha$ and vaccinia growth factor. Science 1987 ; 235 : 350-2.

35. Blistein-Willinger $\mathrm{E}$. The role of growth factors in wound healing. Skin Pharmacol $1991 ; 4: 175-82$.

36. Rothe M, Falanga V. Growth factors. Arch Dermatol $1989 ; 125$ : 1390-8. intracytoplasmique de la chaînc $\beta$ interagit avec la taline, l' $\alpha$-actinine, la vinculine et au travers d'elles le cytosquelette d'actine. Des foyers riches en talinc et en intégrines ont été mis en évidence à la pointe des câbles d'actine au sein des lamellipodes de fibroblastes [11] et dans les points focaux. Ces régions peuvent servir à ancrer les filaments d'actine et à contrôler leur élongation. Les domaines intracytoplasmiques des chaînes $\alpha$, probablement en collaboration avec la sous-unité $\beta$, joueraient un rôle dans la transduction du signal dû à l'interaction intégrine/ligand. Chaque domaine assurcrait un ensemble de fonctions spécifiques, discriminant ainsi l'apparente redondance liée à l'existence dc plusieurs domaines extracellulaires reconnaissant le même substrat. Chan et al. [29] ont ainsi décrit un effet inhibiteur des domaines intracytoplasmiques $\alpha 2$ et $\alpha 5$ sur la motricité de cellules de rhabdomyosarcome. Mais ce phénomène va à l'encontre de l'effet promoteur de VLA2 et VLA5 sur la migration kćratinocytairc rapporté par Kim et al. [30], laissant entrcvoir la possibilité d'unc modulation de réponse selon le type cellulaire.

Les mécanismes au travers desquels le domaine intracytoplasmique contrôle la migration sont inconnus, mais on sait, par excmplc, que la fixation dc certaines intégrines à leur ligand module l'activation de plusieurs phospholipases, la voie des polyphosphatidyl-inositides et la concentration calcique intracytoplasmique pouvant ainsi régler l'activation de molécules comme la gelsolinc. Hanks et al. viennent notamment de démontrer que la FAK (focal adhesion proteintyrosine kinase) pourrait jouer un rôle clé dans la transduction du signal qui fait suite à l'adhérence d'une cellule à son substrat $\left(\mathrm{m} / \mathrm{s} n^{\circ} 2\right.$, vol. 9, p. 141) [31]

Les kératinocytes fraîchement isolés de la peau sont des cellules immobiles ne formant pas de point focaux, n'exprimant ni VLA5 ni $\alpha v \beta 5$ et synthétisant essentiellement des chaînes $\beta 1$ immatures non exprimées à la surface ccllulaire. A la suite d'une plaie, ou après quelques jours de culture, tous ces phénomènes s'inversent et on observe parallèlement une surexpression d' $\alpha \mathrm{v} \beta 5, \alpha 3 \beta 1$ et $\alpha 5 \beta 1$.
Le kératinocytc "activé " pcut alors migrer sur différentes macromolécules qui entrent pour la plupart dans la composition du lit de la plaic. L'anticorps anti- $\alpha 5$ inhibe considćrablement ct spécifiquement la migration sur la fibronectine, ce qui est en accord avec le rôle de récepteur qu'a VLA5 pour cette molécule. Lc mêmc effet est observé avec un anticorps anti- $\alpha 2$ et les collagènes I et IV, or VLA2 les reconnaît tous les dcux ainsi que la laminine. En revanche, si VLA3 cst un récepteur à la fois pour la laminine, l'épiligrine, la fibronectine, les collagènes I et IV, l'utilisation d'un anticorps anti- $\alpha 3$ stimule la migration kératinocytairc sur ces trois dernic̀res molécules. Pour Kim et al. [30], cet effet pourrait être la conséquence de l'inhibition de la formation de points focaux dont on sait que la présence est inverscment proportionnelle à la capacité de migration. Nous avons pour notre part montré que la régulation de la migration de kératinocytes humains normaux in vitro sur du collagène IV ou de la fibronectine ne se faisait pas au travers d'une modulation quantitative de l'expression membranaire des intégrines VLA-2 et VLA-5 [27].

\section{Implications thérapeutiques des données acquises in vitro}

Comprendrc, pour pouvoir les moduler, les mécanismes réglant la migration des kératinocytes est un des défis qu'ont entrepris de relever les dermatologues et les chercheurs travaillant sur la physiologie et la pathologie de la cicatrisation cutanée [32]. De nombreuses ćquipes ont étudié in vivo l'effet dc diverses cytokines sur la cicatrisation cutanée. Citons, à titre d'exemple, le travail de Brown et al. où l'EGF a été testé chez l'homme dans une étude prospective, contrôlée en double aveugle, sur des sites donneurs de greffe [33]. L'EGF recombinant, appliqué deux fois par jour, permettrait d'obtenir une réépithélialisation plus rapide sur les sites traités avec EGF en comparaison avec ceux traités avec un placebo. Une autre étude intéressante, publiée cette fois-ci par Schultz et al. analysait l'effet de TGF $\alpha$ sur la cicatrisa- 
tion chez le porc (brûlures au deuxième degré) [34]. TGF $\alpha$, utilisé à faible dose $(0,1 \mu \mathrm{g} / \mathrm{ml})$, accélérait la régénération épidermique. De plus, les plaies traitées par TGF $\alpha$ présentaient un tissu de granulation plus important que celles traitées par le véhicule seul. Bien d'autres molécules ont été testées in vivo. Le but de cet article n'étant pas d'être exhaustif, le lecteur pourra se reporter à des articles de synthèse pour plus d'informations [35, 36]. La mise en évidence du rôle majeur joué par certaines intégrines dans la fonction migratoire des kératinocytes ouvre la porte à des perspectives thérapeutiques novatrices. Il paraît en effet possible d'agir aussi bien sur leur fonction de récepteur membranaire transmettant un signal spécifique, que sur leur fonction de molécules d'adhérence créant un lien physique entre la ccllule et son support. La détermination des séquences d'acides aminés responsables, au sein des macromolécules matricielles, de l'effet de stimulation ou d'inhibition de la migration kératinocytaire devrait déboucher sur l'apparition de peptides de synthèse utilisables par voie locale pour accélérer le phénomène de cicatrisation

TIRÉS A PART

Y. Sarret.

\section{Summary}

Mechanics and regulation of keratinocyte migration

During cutaneous wound healing, basal keratinocytes migrate laterally to cover the wound bed, whereas in an unwounded situation they move vertically and differentiate to constitute the different layers of the epidermis. Many extracellular factors influence the behavior of these cells. The main ones are extracellular matrix molecules and cytokines present in the dermoepidermal junction and/or in the wound bed. Some of them such as collagens type I and IV, fibronectin and epidermal growth factor are known to induce basal keratinocyte migration. On the contrary, others, such as laminin, inhibit it. Molecules from the VLA-integrin family are receptors for many of these extracellular matrix molecules. As specific adhesion and signal transduction molecules, they play a critical role in keratinocyte migration by allowing interactions between the cell cytoskeleton and the macromolecules in the wound bed. This type of interaction is commonly seen in all the models of cell migration reviewed herein. These models try to explain the different mechanisms involved in basal keratinocyte migration. The translation of this phenomenon, at a light microscopic level is the extension of the lamellipodia/fillopodia at the leading edge of the cell. Finally, we present some clinical applications of the cumulative knowledge published in this field in basic science journals. 